and to unit operations. In regard to the former they referred to the development of new alloys but stressed the importance of scientific comparison and selection. They referred also to the use of special steels for high-temperature and high-pressure reactions in view of the importance of creep at elevated temperatures as well as to the efforts being made to replace nickel at least in part by other metals in a number of special cast irons, and to the attention devoted to the technique of welding and the interest in organic materials such as rubber. Similarly, the authors briefly reviewed developments in heat transfer, filtration, size reduction, distillation, including the use of a true counter-current system throughout the column, evaporation, drying, dust removal and other unit operations, while in conclusion the question of education was discussed and the importance of avoiding uniformity of training was stressed.

Appropriately enough, a paper by Dr. R. Gilmour at a joint meeting with the Institution of Chemical Engineers on "Chemical Engineering in the Fermentation and Distilling Industries" had much to say about the place of art and science in the distillation of whisky, including the effect of air, water, malt and peat on the production of a high-class whisky.

\section{Diffraction and Refraction of Radio Waves}

$\mathrm{T}$ $\mathrm{HE}$ increasing application to various radio purposes of ultra-short electric waves of less than $10 \mathrm{~m}$. in wave-length has given a new stimulus to the problem of calculating the field strength at distances from the transmitter such that the jonosphere is of negligible influence. The solution of the problem of the diffraction of electric waves around the earth was first given on a sound basis by G. N. Watson in 1918, but this analysis involved assumptions which, while perfectly valid for the long and medium waves then in use for radio communication, are not justifiable for the ultra-short waves now being considered. For example, at long wavelengths, the conductivity of the earth may be assumed to be infinitely great for analytical purposes, but as the wave-length is reduced through and below $10 \mathrm{~m}$., the actual value of the conductivity and also the dielectric constant of the soil over which the waves are propagated has an important influence on the field strength at a distance from the souree. Furthermore, the height of the receiver above the earth's surface may no longer be assumed to be a negligibly small fraction of a wave-length, when the latter is only a metre or two.

In a recent paper published by T. I. Eckersley and G. Millington (Phil. Trans. Roy. Soc., A, 237, $273-309$; 1938), a phase-integral method has been applied to the problem of obtaining a formula for calculating the electric field at a distance from a wireless transmitter. This method agrees with Watson's theory in the case already considered by him, but it has the advantage that it more readily permits of extension to the case of finite earth conductivity and of an elevated transmitter and receiver. The authors claim that the phase integral method has a certain generality in exhibiting the solution as one of a general class appropriate to problems of this kind; this feature encourages the hope that other problems of the type may be solved by similar methods. The application of this analysis to the wave propagation problem has also afforded a solution to the case not considered in the original problem, but of considerable practical importance, where there is a gradient of refractive index in the atmosphere above the earth.

The paper to which reference is made above presents the detailed analysis involved in this work in a form which exhibits clearly the physical nature of the problem. Formulæ are derived from which the field strength may be obtained for any wavelength, distance from the transmitter and conductivity of the ground over which the waves travel. Furthermore, the increase of field as the receiving post is raised above the earth's surface is obtained, and by using the reciprocal theorem, the analysis applies as well to the case when the receiver is on the ground and the transmitter is elevated. By combining both cases the effect of having both the transmitter and receiver can be calculated.

A very satisfactory feature of this investigation, which will be much appreciated by those engaged in practical radio communication, is that the various formulæ have already been reduced to the compu. tation of numerical values. A paper by T. L. Eckersley (J. Inst. Elec. Eng., 80, 286-304; 1937) gave a complete set of field strength/distance curves for wave-lengths between 2 and $10 \mathrm{~m}$. and for conductivities corresponding to land and sea. Other curves provide the relationship between fieldstrength and height, while a simple means of extending the curves to take account of refraction through the atmosphere is explained for application when the vertical gradient of refractive index of the air is known or can be estimated. While these theoretical results await adequate experimental confirmation, they are already of considerable value as a general guide to the radio engineer; and in this case the graphs referred to above were utilized by the recent International Telecommunications Conference in Cairo in considering a preliminary allocation of bands of ultra-short waves to various classes of radio communication.

$$
\text { R. L. S.-R. }
$$

\section{University Events}

GLasgow.-On September 29, the Gardiner Institute in Medicine, attached to the Western Infirmary, Glasgow, was opened by Lady Gardiner. The Institute is under the direction of the regius professor of medicine in the University, and will be devoted to clinical research. At the opening ceremony, Prof. T. R. Elliott delivered an address in which he stressed the great advantage to be derived from the establishment of such an institute, and emphasized the neces sity of a closer co-operation between the science and practice of medicine.

The following appointments have recently been made in the University : Dr. O. Löwenstein to be lecturer in zoology in succession to Dr. H. B. Cott, newly appointed lecturer in vertebrate zoology, Cambridge; Mr. Vernon D. van Someren to be temporary assistant in zoology in succession to Dr. Margaret W. Jepps, who has been awarded a Leverhulme fellowship for one year. 